Research Article

\title{
Energy Flow Analysis of One-Dimensional Structures with Random Properties
}

\author{
Kun Wang $\mathbb{D}^{1},{ }^{1}$ Yu Fu, ${ }^{2}$ Jiafu Liu $\mathbb{D}^{3}$ and Qi Zhang ${ }^{1}$ \\ ${ }^{1}$ School of Aeronautics and Astronautics, North China Institute of Aerospace Engineering, Langfang 065000, China \\ ${ }^{2}$ Beijing Institute of Astronautical Systems Engineering, Beijing 100076, China \\ ${ }^{3}$ School of Aeronautics and Astronautics, Sun Yat-Sen University, Guangzhou 510006, China \\ Correspondence should be addressed to Kun Wang; wangkun@nciae.edu.cn and Jiafu Liu; liujiafuericking@163.com
}

Received 19 June 2020; Revised 17 October 2020; Accepted 29 October 2020; Published 8 December 2020

Academic Editor: Giuseppe Ruta

Copyright ( $\odot 2020$ Kun Wang et al. This is an open access article distributed under the Creative Commons Attribution License, which permits unrestricted use, distribution, and reproduction in any medium, provided the original work is properly cited.

In this work, the energy density responses of one-dimensional structures with random properties are investigated analytically. Based on Green kernels, analytical representations of energy density for vibrating rods and beams are proposed using the superposition of energy waves. Considering random properties in rods and beams, formulations of energy density responses are obtained. Then, the mathematical expectations and variances are derived. And response intervals for random responses are developed. Finally, numerical simulations are performed to validate the proposed formulations, and characteristics of the random energy density responses of rods and beams are analysed. The main contribution of this work is that a new approach to energy density responses is proposed which facilitates the vibration analysis of structures with uncertainty parameters.

\section{Introduction}

Prediction of structural vibration is of interest in engineering applications. In low frequencies, the finite element method (FEM) is usually used. However, as the frequency increases, the wavelengths become smaller and more degrees of freedom are desired to obtain accurate results. FEM becomes time-consuming and even unfeasible for many problems. An approach is evaluating the responses with an averaged variable. Thus, statistical energy analysis (SEA) was proposed by Lyon et al. [1-3]. SEA, providing averaged energy for subsystems, reduces the scale of computation dramatically and has been widely studied and applied in various fields [4-8].

However, there are several limitations in SEA, and one that must be noticed is the energy distribution in subsystems, often concerned in many applications, is lost. Therefore, alternative methods become necessary. Energy flow analysis (EFA), introduced by Belov et al. $[9,10]$, is the representative method. In EFA, space- and frequencyaveraged energy density is used as the main variable to reduce the scale of computation. Then, the dynamic behaviour of structures can be described by the control equation of energy density which is analogous to the heat conductivity equation. FEA, who desires much less computation than traditional FEM with displacement being a main variable, allows more detailed results than SEA. To provide a numerical solution for the energy responses, Nefske and Sung [11] applied FEM in solving control equations of energy density for vibrating beams. It is part of evocation of the energy finite element method (EFEM). Developing control equations for basic structures is a prerequisite for other works and is what much work is focused on. Wohlever and Bernhard [12] produced a systematic procedure to obtain energy density control equations of rods and Euler-Bernoulli beams. Bouthier et al. [13, 14] analysed Kirchhoff plates, membranes, and acoustic cavities by EFEM after constructing control equations of energy density. In-plane motions were not concerned before Park et al. [15] formulated the control equation of energy density for inplane shear waves in coupled thin plates. To take into account the effects of rotational inertia and shear distortion, Park and Hong [16] classified the flexural waves 
into two kinds of waves and developed the energy control equations for Timoshenko beams. They further derived the energy control equations for three kinds of far-field propagating waves in Mindlin plates [17]. Considering lateral motion plays a more important role in high-frequency vibration, Han et al. [18] derived the energy control equation for Rayleigh-Love rods. And the energy control equation of Rayleigh-Bishop rods was formulated by taking into account both lateral motion and shear stiffness. Chen et al. [19] derived the energy density and energy intensity of an Euler-Bernoulli beam with constant axial force. Then, the energy density governing equation is established after introducing the relationship between the wavenumber and group velocity.

In practical engineering, objects to analyse consist of many basic structures. The energy transmission relationships between coupled structures become an essential problem. To predict responses for built-up structures, Cho [20] described energy relationships of coupled structures through energy reflection and transmission coefficients. Then, the global matrix can be obtained by using a joint matrix. Bitsie [21] formulated the coupled relationships between acoustic fields and structures through radiation coefficients. The coupled relationships were used in EFEM to compute responses of plates under point excitation, and the results show that energy dissipation is related to the total dissipated power. Zhang et al. [22-24] applied the energy flow method on plates with fluid load, showing that fluid load leads to a different energy density control equation for plates and influences the energy transmission between the plate and its reinforcement beams. Kwon et al. [25] analysed the wave transmission between cylindrical shells and obtained power reflection and transmission coefficients which can be used to predict energy flow for coupled cylindrical thin shells. Lin et al. [26] proposed a junction formulation for the discontinuity problem of energy density at the junction of two beams with stepped thickness. However, most works on transmission relationship neglect wave filtering effects and are only enough accurate when for weak damping effect. Liu et al. developed an alternative approach for energy transmission relationships using the coupling loss factors (CLFs) and validated the approach on coupled beams and coupled plates [27].

To obtain experimental data of energy densities, Navazi et al. [28] proposed a new method for experimental measurement of energy densities of plates. Using the method, kinetic and potential energy densities were obtained by the experiment. It is shown that the experimental results agree well with that from analysis.

Since space- and frequency-averaged variables are adopted to describe dynamic response of structures, there are no mandatory requirements to decrease the size of the elements or increase the order of the interpolating functions as the frequency increases which happens in FEM for numerical computation. EFA demands much less computation power and provides a potential solution to high-frequency problems. However, most works are focused on deterministic structures whose parameters are known precisely. Due to natural uncertainties in practical parameters, deterministic models may produce unacceptable errors. Since totally avoiding uncertainties is impossible, it is meaningful to improve deterministic methods to predict uncertainties in structural vibration. There are many works dealing with uncertainties in structures [29-33]. Among them, the probabilistic method is preferable. In probabilistic analysis, parameters are supposed to satisfy a certain probabilistic distribution, and responses are provided in probabilistic form by random variables. The responses subject to a certain probabilistic distribution are supposed to be more feasible than deterministic results. In this work, new analytical formulations of energy density responses of vibrating rods and beams are developed with Green kernels. The analytical formulations facilitate the analysis of structural vibration from the point of view of energy. Energy densities can be obtained directly without solving control equations using numerical solutions. Then, random energy density responses are derived for rods and beams with probabilistic parameters. Considering the excitation location as a probabilistic variable, numerical simulations are performed to validate the derived analytical solutions and the characteristics of random response are analysed comparing with deterministic results. The analytical formulations provide helpful representations for further research on energy densities of structures. And for practical structures whose uncertainties should not be neglected, the probabilistic formulations of energy density responses provide a potential approach for the analysis of vibration in high frequencies.

\section{Materials and Methods}

2.1. Energy Density Governing Equation and Numerical Solution. The energy density control equation for rods and beams is as follows [12]:

$$
\frac{c_{g}^{2}}{\eta \omega} \frac{\mathrm{d}^{2} E_{e}}{\mathrm{~d} x^{2}}-\eta \omega E_{e}+\pi_{i n}=0,
$$

where $E_{e}$ is the energy density, $c_{g}$ is the group velocity of waves, $\eta$ is the material damping coefficient, and $\pi_{i n}$ is the input power by the excitation with the frequency $\omega$. To obtain the energy density responses, the control equation can be solved by the finite element method. The discrete control equation becomes

$$
\left[K^{e}\right]\left\{E^{e}\right\}=\left\{F^{e}\right\}+\left\{Q^{e}\right\} .
$$

In equation (2),

$$
\left[K^{e}\right]=\int\left(\frac{c_{g}^{2}}{\eta \omega} \frac{\partial N^{T}}{\partial x} \frac{\partial N}{\partial x}+\eta \omega N^{T} N\right) \mathrm{d} D
$$

is the coefficient matrix,

$$
\left\{F^{e}\right\}=\int N^{T} \pi_{i n} \mathrm{~d} D,
$$

indicates the input power, and 


$$
\left\{Q^{e}\right\}=\int \frac{c_{g}^{2}}{\eta \omega} N^{T}(-n) \frac{\mathrm{d} E_{e}}{\mathrm{~d} x} \mathrm{~d} \Gamma,
$$

is the power flow in elements. The boundary of elements is denoted by $\Gamma$, and the normal vector of the boundary is denoted by $n$.

2.2. Analytical Formulations of Energy Density. Equation (2) provides the numerical form for the calculation of the energy density responses which is suitable for complex structures. However, it is less convenient for studying the characteristics of energy flow in structures than analytical forms. To obtain analytical solutions, one point should be noticed is that energy density responses are reverberant in high frequencies, so the vibration power of a single wave is totally reflected at free and clamped boundaries regardless of the phase. Then, the responses are the summation of a series of energy waves.

2.3. Analytical Solutions for Rods. The control equation of motion for a uniform longitudinally vibrating rod is as follows [12]:

$$
E_{c} S \frac{\partial^{2} w}{\partial x^{2}}+\omega^{2} \rho S w=F \delta\left(x-x_{0}\right) e^{j \omega t} .
$$

In the equation, the coordinate system in Figure 1 is adopted, $w$ is the longitudinal displacement, $S$ is the crosssectional area, $\rho S$ indicates the mass density per unit length, and $F \delta\left(x-x_{0}\right) e^{j \omega t}$ is the harmonic point force applied at location $x-x_{0}$. The damping effect is considered by introducing the complex modulus of elasticity $E_{c}=E(1+j \eta)$, where $\eta$ is the hysteretic damping coefficient and $j=\sqrt{-1}$.

Using the wavenumber, we obtain

$$
\frac{\partial^{2} w}{\partial x^{2}}+k^{2} \frac{\partial \mathrm{w}}{\partial \mathrm{x}}=\frac{F \delta\left(x-x_{0}\right)}{E_{c} S},
$$

where

$$
k=\left(\frac{\omega^{2} \rho}{E(1+j \eta)}\right)
$$

is a complex wavenumber. In lightly damped rods, i.e., $\eta \ll 1$, the wavenumber can be approximated as

$$
k=k_{1}+k_{2},
$$

where $k_{1}=\sqrt{\omega^{2} \rho / E}$ and $k_{2}=-\eta k_{1} / 2$.

The Green kernel, which indicates the displacements of the rod under a unit point force, for a longitudinally vibrating rod with infinite length is as follows [34]:

$$
G_{r}=\frac{1}{j E S k} e^{-j k x_{d}},
$$

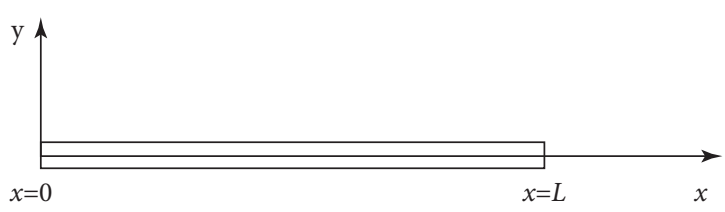

Figure 1: The coordinate system for a rod or beam.

where $x_{d}$ indicates the distance between the response point and the excitation.

The total energy of a vibrating rod includes two parts: the potential energy and the kinetic energy. The time-averaged kinetic $(T)$ and potential $(V)$ energy densities are, respectively, [12] as follows:

$$
\begin{gathered}
T=\frac{1}{2} \rho S\left(\frac{\partial w}{\partial t}\right)^{2}, \\
V=\frac{1}{2} E S\left(\frac{\partial w}{\partial x}\right)^{2} .
\end{gathered}
$$

The averaged complex forms can be written as

$$
\begin{aligned}
& T=\frac{1}{2} \rho S\left(\frac{\partial w}{\partial t}\right)\left(\frac{\partial w}{\partial t}\right)^{*}, \\
& V=\frac{1}{2} E S\left(\frac{\partial w}{\partial x}\right)\left(\frac{\partial w}{\partial x}\right)^{*},
\end{aligned}
$$

in which the star * indicates the conjugation.

Substituting the Green kernel into equations (12) and (13), the averaged kinetic and potential energy densities become

$$
\begin{aligned}
T & =\frac{1}{4} \frac{\rho S \omega^{2}}{\left(E S k_{1}\right)^{2}} e^{2 k_{2} x_{d}}, \\
V & =\frac{1}{4} \frac{1}{E S} e^{2 k_{2} x_{d}},
\end{aligned}
$$

by taking $|k| \approx k_{1}$. Thus, noticing $k_{1}=\sqrt{\omega^{2} \rho / E}$, the energy density responses of an infinite rod under a unit point force are

$$
G_{\mathrm{er}}=T+V=\frac{1}{2} \frac{\rho S \omega^{2}}{\left(E S k_{1}\right)^{2}} e^{2 k_{2} x_{d}} .
$$

The representation for an infinite rod under a sinusoidal force with amplitude $F$ is then written as

$$
E_{\text {er }}=\frac{F^{2}}{2} \frac{\rho S \omega^{2}}{\left(E S k_{1}\right)^{2}} e^{2 k_{2} x_{d}} .
$$

To obtain the representation for a finite rod, the boundary effect should be taken into account. Since what 
exists in a vibrating rod is a reverberant field, power flow is considered to be reflected totally at boundaries regardless of the phase. Then, the response is the summation of a series of energy waves, which can be written as

$$
\begin{aligned}
E_{\mathrm{er}}=\frac{F^{2}}{2} & \frac{\rho S \omega^{2}}{\left(E S k_{1}\right)^{2}}\left(\sum_{n=0}^{N} e^{2 k_{2}\left(x_{d}+2 n L\right)}\right. \\
& \left.+\sum_{n=0}^{N} e^{2 k_{2}\left(x_{d}+2(L-x)+2 n L\right)}\right),
\end{aligned}
$$

where $N$ is the order of reflection, $L$ is the length of the rod, and $x$ indicates the location of the concerned location. As the order of reflection tends to infinite, the representation of the energy density responses is

$$
E_{\mathrm{er}}=\frac{F^{2}}{2} \frac{\rho S \omega^{2}}{\left(E S k_{1}\right)^{2}} \frac{e^{2 k_{2} x_{d}}}{1-e^{4 k_{2} L}}\left(1+e^{4 k_{2}(L-x)}\right) .
$$

Then, the energy density Green kernel for a finite rod can be written as

$$
G_{\mathrm{er}}=\frac{\rho S \omega^{2}}{2\left(E S k_{1}\right)^{2}} \frac{e^{2 k_{2} x_{d}}}{1-e^{4 k_{2} L}}\left(1+e^{4 k_{2}(L-x)}\right) .
$$

2.4. Analytical Solutions for Beams. The control equation of motion for a uniform Euler-Bernoulli beam excited by a transverse harmonic point force is as follows [12]:

$$
E_{c} I \frac{\partial^{4} w}{\partial x^{4}}+\rho S \frac{\partial^{2} w}{\partial x^{2}}=F \delta\left(x-x_{0}\right) e^{j \omega t}
$$

In the equation, $w$ is the transverse displacement, $S$ is the cross-sectional area, $\rho S$ is the mass density per unit length, $F \delta\left(x-x_{0}\right) e^{j \omega t}$ is the harmonic point force applied at location $x_{0}$, the complex modulus of elasticity is $E_{c}=\mathrm{E}(1+j \eta)$, and $E_{c} I$ indicates the flexural rigidity.

The wavenumber $k$ of the bending beam is

$$
k=\left(\frac{\omega^{2} \rho S}{E_{c} I(1+j \eta)}\right)^{1 / 4} \text {. }
$$

In lightly damped beams, the wavenumber can be written as

$$
k=k_{1}+j k_{2}
$$

where $k_{1}=\sqrt{\omega^{2} \rho / E}$ and $k_{2}=-\eta k_{1} / 4$.

The Green kernel for an infinite beam is as follows [35]:

$$
G_{b}=-\frac{1}{4 E_{c} I k^{3}}\left(j e^{-j k x_{d}}+e^{-k x_{d}}\right) .
$$

Similar to a rod, the average total energy density in a beam is the sum of the kinetic and potential energy density:

$$
\begin{aligned}
& T=\frac{1}{2} \rho S\left(\frac{\partial w}{\partial t}\right)\left(\frac{\partial w}{\partial t}\right)^{*}, \\
& V=\frac{1}{2} \operatorname{EI}\left(\frac{\partial w}{\partial x}\right)\left(\frac{\partial w}{\partial x}\right)^{*},
\end{aligned}
$$

where the star * indicates the conjugation.

Substituting the displacement with the Green kernel, the energy densities become

$$
\begin{aligned}
T & =\frac{F^{2} \rho S \omega^{2}}{16\left(E_{c} I\right)^{2} k^{6}}\left(e^{2 k_{2} x_{d}}+j e^{-\left(j k+k^{*}\right) x_{d}}-j e^{\left(j k^{*}-k\right) x_{d}}+e^{-2 k_{1} x_{d}}\right), \\
V & =\frac{F^{2}}{16 E_{c} I k^{4}}\left(e^{2 k_{2} x_{d}}+e^{-\left(j k+k^{*}\right) x_{d}}+e^{-\left(j k^{*}+k\right) x_{d}}+e^{-2 k_{1} x_{d}}\right) .
\end{aligned}
$$

Based on the approximation $k_{1}-k_{2} \approx k_{1}$, the energy density representation for an infinite Euler-Bernoulli beam

$$
E_{\mathrm{eb}}=\frac{F^{2}}{8} \frac{\rho S \omega^{2}}{\left(E_{c} I\right)^{2} k^{6}} e^{2 k_{2} x_{d}}
$$

can be adopted without introducing unacceptable errors by noticing that $k_{2} \ll k_{1}$.

The energy density responses for a finite beam are calculated by the superposition of a series of bending waves:

$$
\begin{array}{r}
E_{\mathrm{eb}}=\frac{F^{2}}{8} \frac{\rho S \omega^{2}}{\left(E_{c} I\right)^{2} k_{1}^{6}}\left(\sum_{n=0}^{N} e^{2 k_{2}\left(x_{d}+2 n L\right)}+\sum_{n=0}^{N} e^{2 k_{2}\left(x_{d}+2(L-x)+2 n L\right)}\right), \\
E_{\mathrm{eb}}=\frac{F^{2}}{8} \frac{\rho S \omega^{2}}{\left(E_{c} I\right)^{2} k_{1}^{6}} \frac{e^{2 k_{2} x_{d}}}{1-e^{4 k_{2} L}}\left(1+e^{4 k_{2}(L-x)}\right)
\end{array}
$$

Then, the energy density Green kernel for a finite rod can be written as

$$
G_{\mathrm{eb}}=\frac{\rho S \omega^{2}}{8\left(E_{c} I\right)^{2} k_{1}^{6}} \frac{e^{2 k_{2} x_{d}}}{1-e^{4 k_{2} L}}\left(1+e^{4 k_{2}(L-x)}\right) .
$$


It can be observed that excitation terms are introduced in equations (18) and (27). The effect of boundaries is taken into account by the sum of reflected waves. The expressions become analytical forms which have no unknown constants, and it is convenient for analysing the energy density characteristics of rods and beams.

\section{Energy Densities for One-Dimensional Structures with Random Uncertainties}

3.1. Energy Densities for Rods with Random Uncertainties. According to equation (18), the energy density formulation of vibrating rods with random parameters can be written as

$$
\widetilde{E}_{\mathrm{er}}=\frac{F^{2}}{2} \frac{\widetilde{\rho} \widetilde{S} \omega^{2}}{\left(\widetilde{E} \widetilde{S} k_{1}\right)^{2}} \frac{e^{2 k_{2} \tilde{x}_{d}}}{1-e^{4 k_{2} \widetilde{L}}}\left(1+e^{4 k_{2}(\widetilde{L}-x)}\right),
$$

where $\widetilde{a}$ denotes an independent random variable with the mathematic expectation $\bar{a}$ and the standard deviation $\sigma_{a}$.

Then, the expectation of the response is

$$
\bar{E}_{\mathrm{er}}=\mathbf{E}_{\mathrm{er}}+\operatorname{EXP}\left(\sum_{a=\rho, E, S, L, x_{d}} \frac{\partial \mathbf{E}_{\mathrm{er}}}{\partial a} \delta a\right),
$$

in which EXP means expectation, and $\mathbf{E}_{\mathrm{er}}$ indicates the function which possesses the same form as the deterministic formulation of energy densities:

$$
\begin{aligned}
& \mathbf{E}_{\mathrm{er}}=E_{\mathrm{er}}=\frac{F^{2}}{2} \frac{\rho S \omega^{2}}{\left(E S k_{1}\right)^{2}} \frac{e^{2 k_{2} x_{d}}}{1-e^{4 k_{2} L}}\left(1+e^{4 k_{2}(L-x)}\right), \\
& \delta a=\tilde{a}-\bar{a} .
\end{aligned}
$$

The variance of response is obtained as

$$
\sigma_{\mathrm{er}}^{2}=\sum_{a=\rho, E, S, L, x_{d}}\left(\frac{\partial \mathbf{E}_{\mathrm{er}}}{\partial a} \sigma_{a}\right)^{2} .
$$

And the standard deviation is

$$
\sigma_{\mathrm{er}}^{2}=\sqrt{\sum_{a=\rho, E, S, L, x_{d}}\left(\frac{\partial \mathbf{E}_{\mathrm{er}}}{\partial a} \sigma_{a}\right)^{2}} .
$$

As an example, the energy density of vibrating rods experiencing a random-location excitation can be written as

$$
\widetilde{E}_{\mathrm{er}}=\frac{F^{2}}{2} \frac{\rho S \omega^{2}}{\left(\mathrm{ES} k_{1}\right)^{2}} \frac{e^{2 k_{2} \tilde{x}_{d}}}{1-e^{4 k_{2} L}}\left(1+e^{4 k_{2}(L-x)}\right) .
$$

The mathematic expectation and variance of the random variable $\tilde{x}_{d}$ are

$$
\begin{aligned}
\operatorname{EXP}\left(\tilde{x}_{d}\right) & =x_{d}, \\
\operatorname{var}\left(\tilde{x}_{d}\right) & =\sigma_{x_{d}}^{2} .
\end{aligned}
$$

Equation (34) can be expanded as a Taylor series at $x_{d}$, and the first two terms are retained as

$$
\begin{aligned}
\widetilde{E}_{\mathrm{er}}= & \frac{F^{2}}{2} \frac{\rho S \omega^{2}}{\left(E S k_{1}\right)^{2}} \frac{e^{2 k_{2} \tilde{x}_{d}}}{1-e^{4 k_{2} L}}\left(1+e^{4 k_{2}(L-x)}\right) \\
& \cdot\left(1+2 k_{2}\left(\widetilde{x}_{d}-x_{d}\right)\right) .
\end{aligned}
$$

Then, assuming the random location as a Gaussian random parameter, the expectation of energy density for rods is as

$$
\bar{E}_{\mathrm{er}}=\frac{F^{2}}{2} \frac{\rho S \omega^{2}}{\left(E S k_{1}\right)^{2}} \frac{e^{2 k_{2} x_{d}}}{1-e^{4 k_{2} L}}\left(1+e^{4 k_{2}(L-x)}\right),
$$

with

$$
\operatorname{mean}\left(\tilde{x}_{d}-x_{d}\right)=0 \text {. }
$$

It is the same expression as that of rods experiencing a deterministic-location excitation at $x=x_{d}$. Thus, the standard deviation of the energy density responses is

$$
\sigma_{\mathrm{er}}=-\frac{F^{2}}{2} \frac{\rho S \omega^{2}}{\left(\mathrm{ES} k_{1}\right)^{2}} \frac{2 k_{2} \sigma_{x_{d}} e^{2 k_{2} x_{d}}}{1-e^{4 k_{2} L}}\left(1+e^{4 k_{2}(L-x)}\right) \text {. }
$$

Within $n$ standard deviations, the corresponding energy density interval is

$$
E_{\mathrm{er}}^{I}=\left[\bar{E}_{\mathrm{er}}-n \sigma_{\mathrm{er}}, \bar{E}_{\mathrm{er}}+n \sigma_{\mathrm{er}}\right],
$$

in which at least $1-\left(1 / n^{2}\right)$ of the values of response are covered. The maximum and minimum values in the interval are

$$
\begin{aligned}
& E_{\text {ermax }}=\frac{F^{2}}{2} \frac{\rho S \omega^{2}}{\left(E S k_{1}\right)^{2}} \frac{1-2 n k_{2} \sigma_{x_{d}}}{1-e^{4 k_{2} L}}\left(1+e^{4 k_{2}(L-x)}\right) e^{2 k_{2} x_{d}}, \\
& E_{\text {ermax }}=\frac{F^{2}}{2} \frac{\rho S \omega^{2}}{\left(E S k_{1}\right)^{2}} \frac{1+2 n k_{2} \sigma_{x_{d}}}{1-e^{4 k_{2} L}}\left(1+e^{4 k_{2}(L-x)}\right) e^{2 k_{2} x_{d}} .
\end{aligned}
$$

3.2. Energy Density for Beams with Random Uncertainties. For an Euler-Bernoulli vibrating beam with random parameters, the energy density representation is

$$
E_{\mathrm{eb}}=\frac{F^{2}}{4} \frac{\tilde{\rho} \widetilde{S} \omega^{2}}{\left(\widetilde{E}_{c} I\right)^{2} k_{1}^{6}} \frac{e^{2 k_{2} \tilde{x}_{d}}}{1-e^{4 k_{2} \widetilde{L}}}\left(1+e^{4 k_{2}(\widetilde{L}-x)}\right) .
$$

Then, the expectation of responses is similarly

$$
\bar{E}_{\mathrm{eb}}=\mathbf{E}_{\mathrm{eb}}+\operatorname{EXP}\left(\sum_{a=\rho, E, S, L, x_{d}} \frac{\partial \mathbf{E}_{\mathrm{eb}}}{\partial a} \delta a\right),
$$

in which

$$
\begin{aligned}
& \mathbf{E}_{\mathrm{eb}}=E_{\mathrm{eb}}=\frac{F^{2}}{4} \frac{\rho S \omega^{2}}{\left(E_{c} I\right)^{2} k_{1}^{6}} \frac{e^{2 k_{2} x_{d}}}{1-e^{4 k_{2} L}}\left(1+e^{4 k_{2}(L-x)}\right), \\
& \delta a=\tilde{a}-a .
\end{aligned}
$$


The variance of response is obtained as

$$
\sigma_{\mathrm{eb}}^{2}=\sum_{a=\rho, E, S, L, x_{d}}\left(\frac{\partial \mathbf{E}_{\mathrm{eb}}}{\partial a} \sigma_{a}\right)^{2} .
$$

And the standard deviation is

$$
\sigma_{\mathrm{er}}^{2}=\sqrt{\sum_{a=\rho, E, S, L, x_{d}}\left(\frac{\partial \mathbf{E}_{\mathrm{er}}}{\partial a} \sigma_{a}\right)^{2}} .
$$

Then, for a vibrating beam experiencing random-location excitation, the energy density is

$$
\widetilde{E}_{\mathrm{eb}}=\frac{F^{2}}{4} \frac{\rho S \omega^{2}}{\left(E_{c} I\right)^{2} k_{1}^{6}} \frac{e^{2 k_{2} \tilde{x}_{d}}}{1-e^{4 k_{2} L}}\left(1+e^{4 k_{2}(L-x)}\right) .
$$

The mathematic expectation and the variance of the random variable $\tilde{x}_{d}$ are given as

$$
\begin{gathered}
\operatorname{EXP}\left(\tilde{x}_{d}\right)=x_{d}, \\
\operatorname{var}\left(\tilde{x}_{d}\right)=\sigma_{x_{d}}^{2} .
\end{gathered}
$$

The first two terms of the Taylor series at $x_{d}$ are retained. The energy density formulation is approximately written as

$$
\begin{gathered}
\widetilde{E}_{\mathrm{eb}}=\frac{F^{2}}{4} \frac{\rho S \omega^{2}}{\left(E_{c} I\right)^{2} k_{1}^{6}} \frac{e^{2 k_{2} x_{d}}}{1-e^{4 k_{2} L}}\left(1+e^{4 k_{2}(L-x)}\right) \\
\cdot\left(1+2 k_{2}\left(\tilde{x}_{d}-x_{d}\right)\right) .
\end{gathered}
$$

Then, the expectation and the standard deviation of the energy densities can be obtained by assuming the location as a Gaussian random parameter:

$$
\begin{aligned}
& \bar{E}_{\mathrm{eb}}=\frac{F^{2}}{4} \frac{\rho S \omega^{2}}{\left(E_{c} I\right)^{2} k_{1}^{6}} \frac{e^{2 k_{2} x_{d}}}{1-e^{4 k_{2} L}}\left(1+e^{4 k_{2}(L-x)}\right), \\
& \sigma_{\mathrm{eb}}=-\frac{F^{2}}{4} \frac{\rho S \omega^{2}}{\left(E_{c} I\right)^{2} k_{1}^{6}} \frac{2 k_{2} \sigma_{x_{d}} e^{2 k_{2} x_{d}}}{1-e^{4 k_{2} L}}\left(1+e^{4 k_{2}(L-x)}\right) .
\end{aligned}
$$

Within $n$ standard deviations, the corresponding energy density interval is

$$
E_{\mathrm{eb}}^{I}=\left[\bar{E}_{\mathrm{eb}}-n \sigma_{\mathrm{eb}}, \bar{E}_{\mathrm{eb}}+n \sigma_{\mathrm{er}}\right],
$$

in which at least $1-\left(1 / n^{2}\right)$ of the values of response are covered. Maximum and minimum energy densities in the interval for beams are

$$
\begin{aligned}
& E_{\mathrm{eb}}=\frac{F^{2}}{4} \frac{\rho S \omega^{2}}{\left(E_{c} I\right)^{2} k_{1}^{6}} \frac{\left(1-2 n k_{2} \sigma_{x_{d}}\right)}{1-e^{4 k_{2} L}}\left(1+e^{4 k_{2}(L-x)}\right) e^{2 k_{2} x_{d}}, \\
& E_{\mathrm{eb}}=\frac{F^{2}}{4} \frac{\rho S \omega^{2}}{\left(E_{c} I\right)^{2} k_{1}^{6}} \frac{\left(1+2 n k_{2} \sigma_{x_{d}}\right)}{1-e^{4 k_{2} L}}\left(1+e^{4 k_{2}(L-x)}\right) e^{2 k_{2} x_{d}} .
\end{aligned}
$$

\section{Results and Discussion}

4.1. A Simulation for the Rod. For the rod cases, the uniform clamped-free rod shown in Figure 2 with $\rho S=200 \mathrm{~kg} / \mathrm{m}$, $E=7.1 \times 10^{5} \mathrm{~Pa}, L=1 \mathrm{~m}$, and $\eta=0.02$ is driven by a harmonic point force with $\omega=0 \sim 1000 \mathrm{rad} / \mathrm{s}$. Figure 3 shows the analytical energy density distribution in frequencies at location $x=L / 2$ with that obtained from EFEM and wave methods. And the distributions in space at $\omega=872 \mathrm{rad} / \mathrm{s}$ are presented in Figure 4.

In Figures 3 and 4, it can be observed that the proposed analytical solution to the rod coincides well with the numerical (EFEM) solution of the energy density governing equation. Both of them represent well the global variation in the classical wave solution regardless of the excitation frequency.

Figure 5 shows the energy density interval at point $x=L / 2$ in frequencies by assuming the excitation coordinate as a Gaussian random variable and the standard deviation $\sigma=0.1 \mathrm{~L}$. Due to the random effect of the excitation location, the energy densities are no longer deterministic. A confidence response interval is generated by potential energy densities for both frequency and space responses. The practical responses can be any value in the interval. However, the mean energy densities are equal to the results from deterministic parameters without deviations. As the excitation frequency increases, the energy density interval width increases. And it is indicated that the uncertainty of energy densities for the rod becomes more significant in high frequencies than in low frequencies.

The random energy densities in space at $\omega=872 \mathrm{rad} / \mathrm{s}$ are shown in Figure 6. The mean energy densities are identical to the results from deterministic parameters without uncertainties. The energy density interval width remains with increasing distance from the driving point which indicates that energy densities of the rod in different locations possesses the same uncertainty at a fixed frequency.

The standard deviations and the relative standard deviations of energy densities are presented, respectively, in Figures 7 and 8. It is shown that the standard deviation at the free end of the rod increases while that at the clamped end decreases as the frequency increases. The relative standard deviation of energy density increases as the frequency increases, and it stays the same with increasing distance from the driving point. It is indicated that, although the absolute uncertainty changes in various ways in different locations, the relative uncertainty of energy densities for the rod increases in high frequencies and remains at a steady level at a fixed frequency regardless of the coordination.

4.2. A Simulation for the Beam. The beam used for the validation is shown in Figure 9 with $L=5 \mathrm{~m}$, $\mathrm{EI}=7200(1+j \eta) \mathrm{Nm}^{2}, \eta=0.01$, and $\rho S=200 \mathrm{~kg} / \mathrm{m}$. Figure 10 provides the energy density distributions in frequencies at $x=L / 2$ from the proposed analytical, the numerical (EFEM), and the wave methods. And the energy density distributions in space at $\omega=973 \mathrm{rad} / \mathrm{s}$ are shown in 


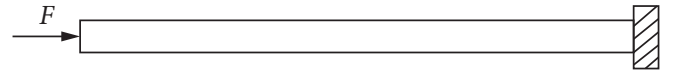

Figure 2: A free-clamped vibrating rod.

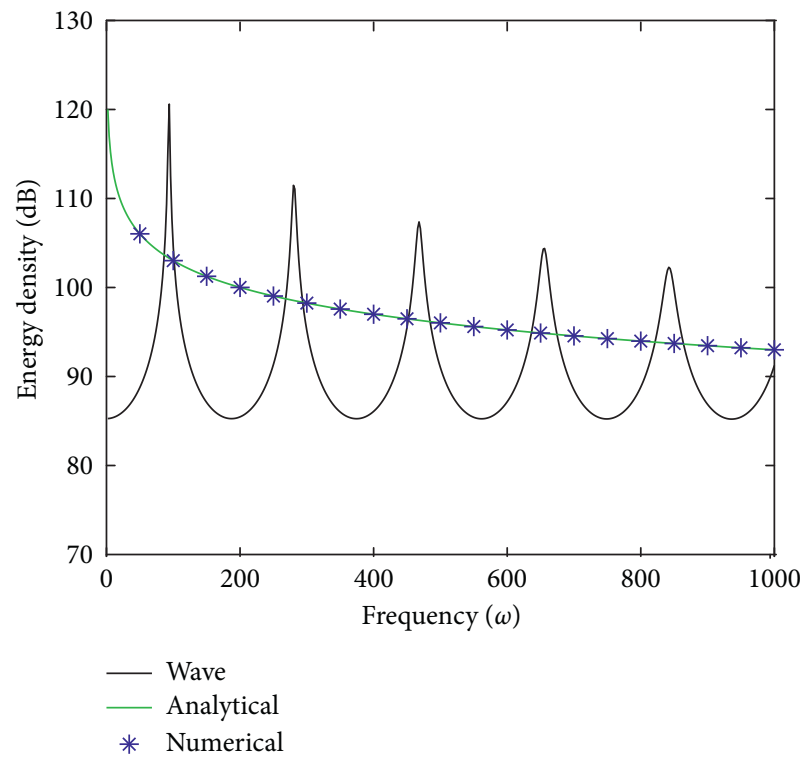

Figure 3: Energy densities of the rod in frequency field. The reference energy density is $10^{-12} \mathrm{~J} / \mathrm{m}^{2}$.

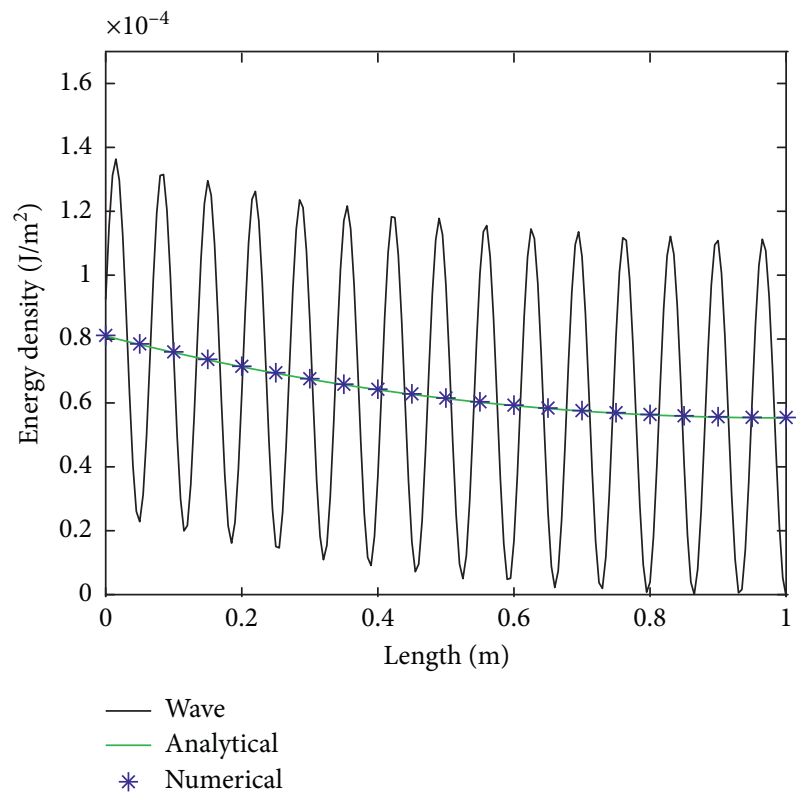

Figure 4: Energy densities of the rod in space.

Figure 11. It is observed that, for the beam, results from the proposed analytical method and the numerical method coincide well and represent well the global variation in the classical wave solution regardless of the excitation frequency.

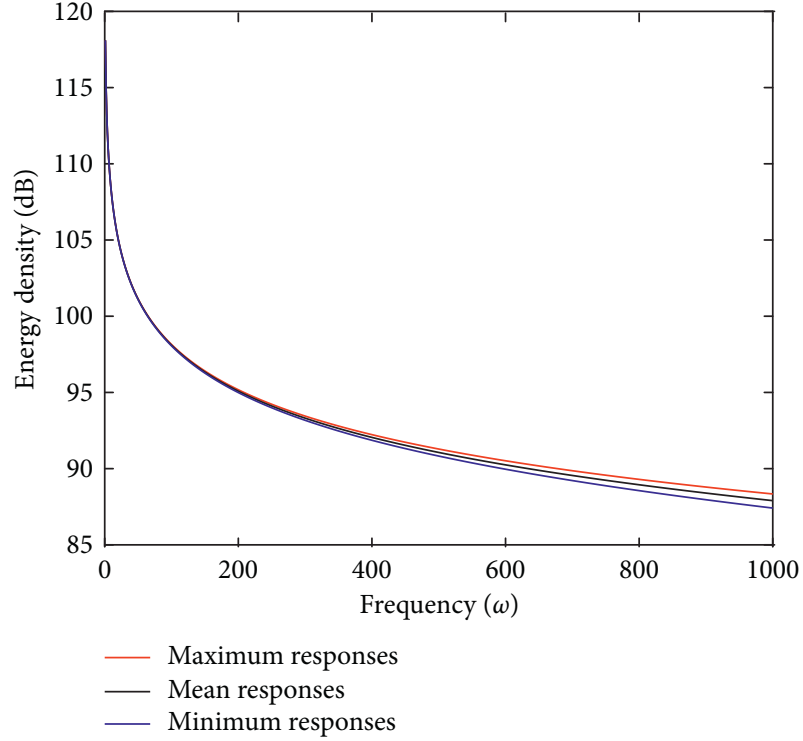

Figure 5: Energy density interval of the rod with a random-location excitation in frequency field. The reference energy density is $10^{-12} \mathrm{~J} / \mathrm{m}^{2}$.

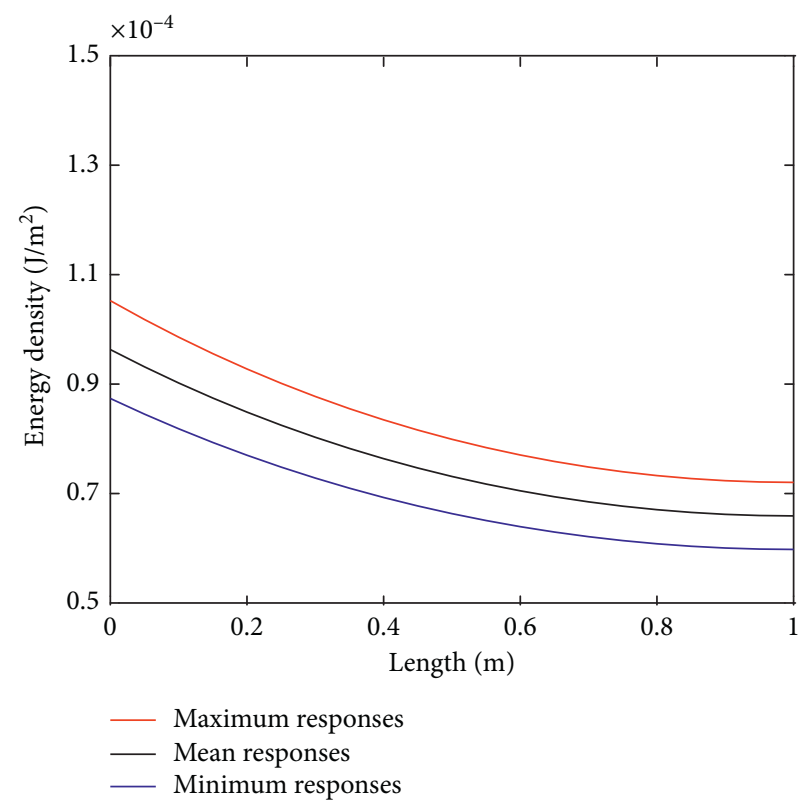

Figure 6: Energy density interval of the rod with a random-location excitation in space.

Similar to the previous example for the rod, Figure 12 shows the energy density responses of the beam at point $x=L / 2$ in the case of the excitation location standard deviation $\sigma=0.2 \mathrm{~L}$. Due to the random effect of the excitation location, the energy densities of the beam become random. And the potential responses cover an interval in the frequency domain and spatial domain. And the mean energy densities are equal to the results from deterministic parameters without deviations. As the excitation frequency increases, the uncertainty of energy density 


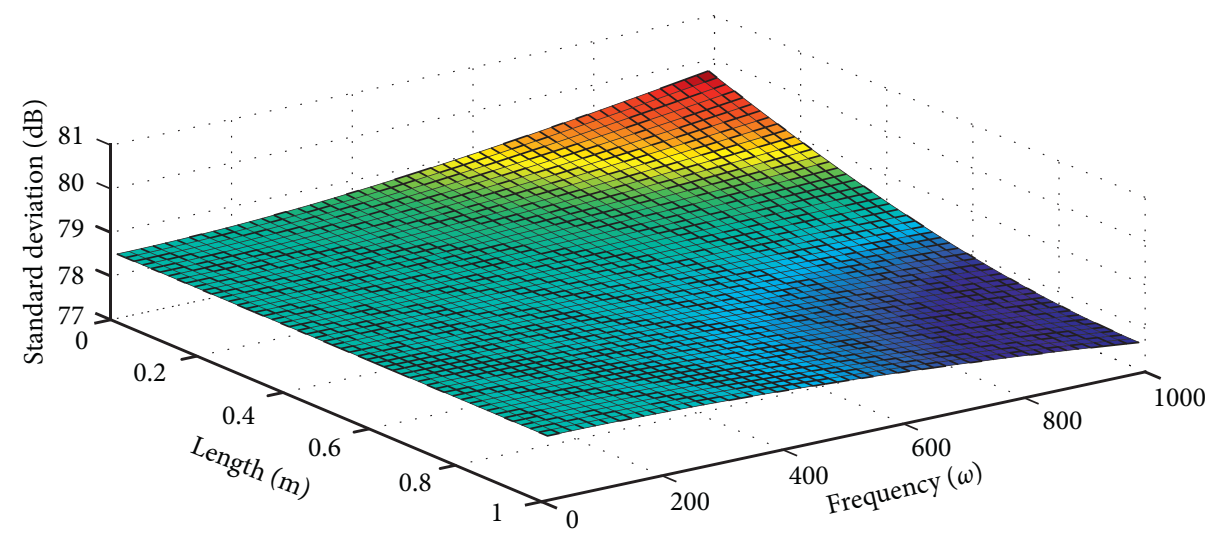

Figure 7: Standard deviations of energy densities of the rod. The reference energy density is $10^{-12} \mathrm{~J} / \mathrm{m}^{2}$.

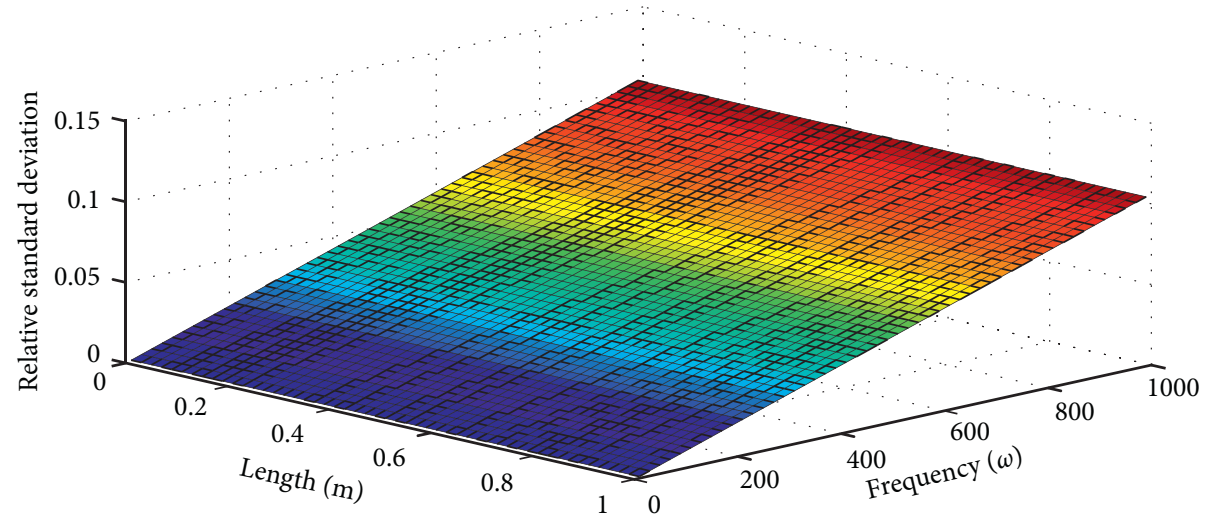

Figure 8: Relative standard deviations of energy densities of the rod.

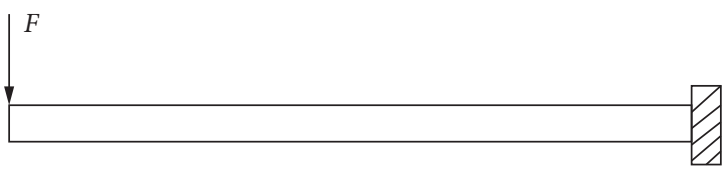

Figure 9: A cantilever beam.

for the beam becomes more significant. Figure 13 shows the energy densities in space at $\omega=973 \mathrm{rad} / \mathrm{s}$ whose potential values form an interval too. The mean energy densities are identical to the results from deterministic parameters without uncertainties. And the energy densities of the beam in different locations possess the same uncertainty at a fixed frequency.

The standard deviation and the relative standard deviation of energy densities are presented, respectively, in Figures 14 and 15. It is observed that the standard deviation is shown to decrease with the increasing frequency at all locations of the beam. The relative standard deviation increases with the increasing frequency at all locations of the beam and stays the same with increasing distance from the driving point. It is indicated although the absolute uncertainty goes down, the relative uncertainty of energy densities

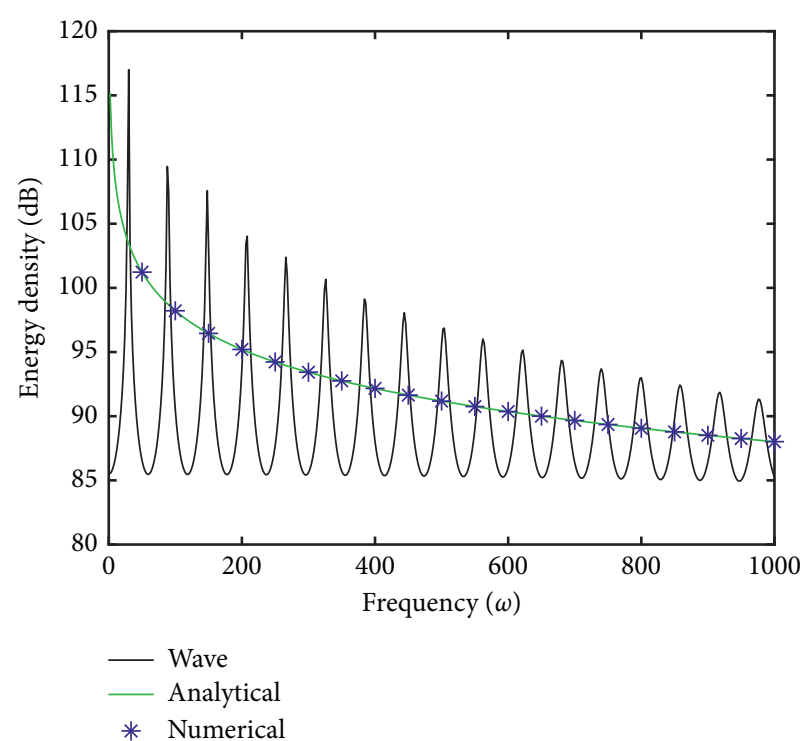

Figure 10: Energy densities of the beam in the frequency field. The reference energy density is $10^{-12} \mathrm{~J} / \mathrm{m}^{2}$. 


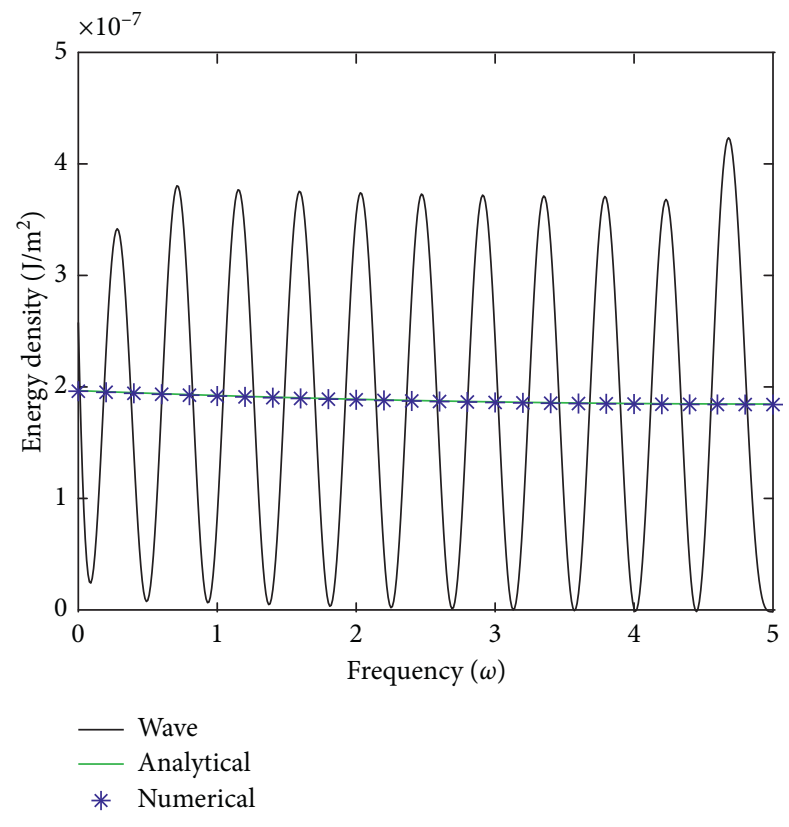

FIgURE 11: Energy densities of the beam in space.

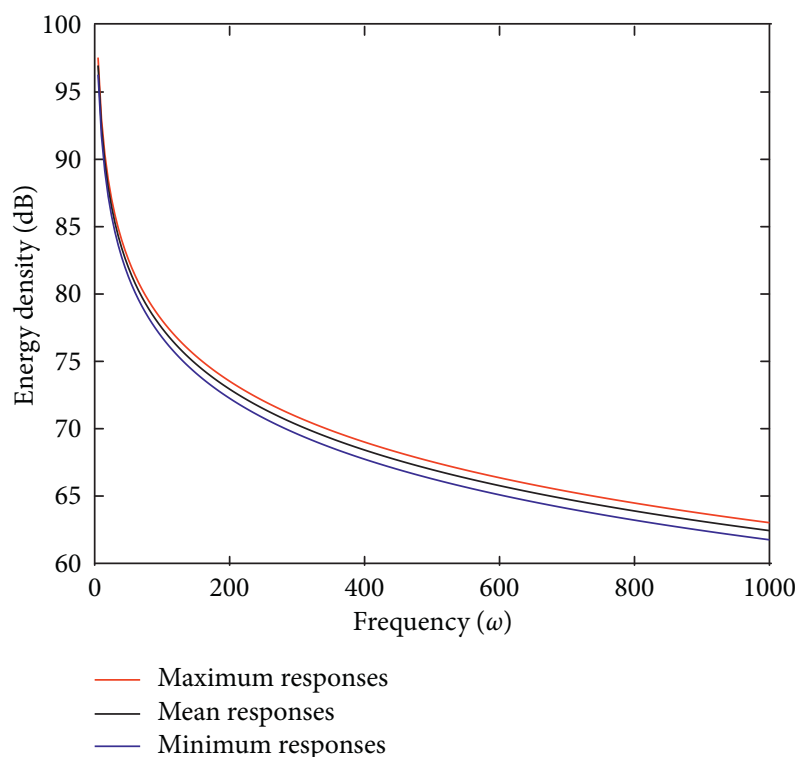

Figure 12: Energy density interval of the beam with a random-location excitation in the frequency field. The reference energy density is $10^{-12} \mathrm{~J} / \mathrm{m}^{2}$. 


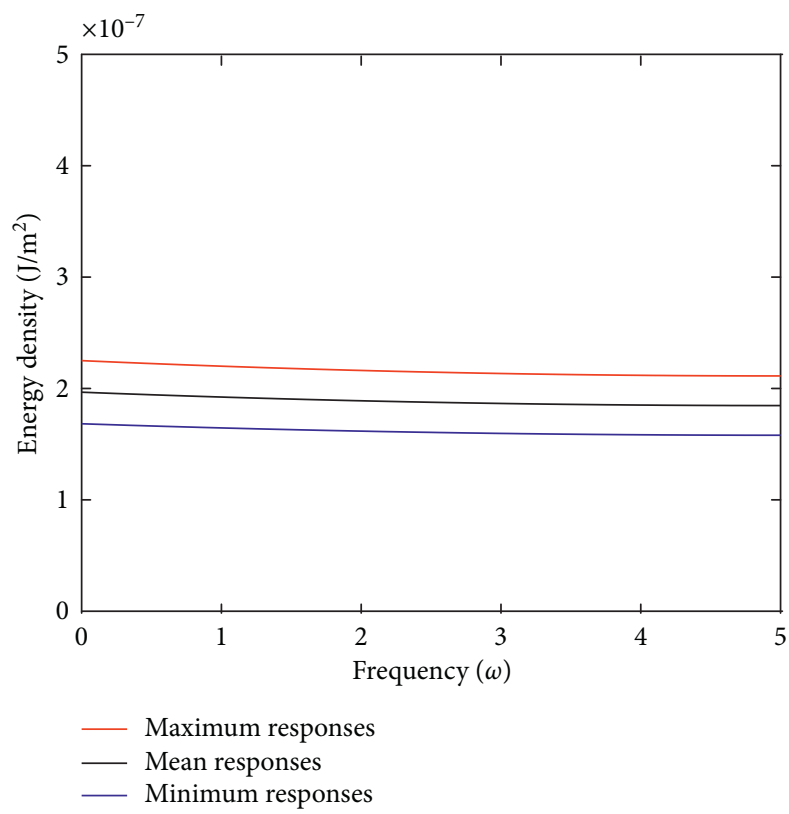

FIGURE 13: Energy density interval of the beam with a random-location excitation in space.

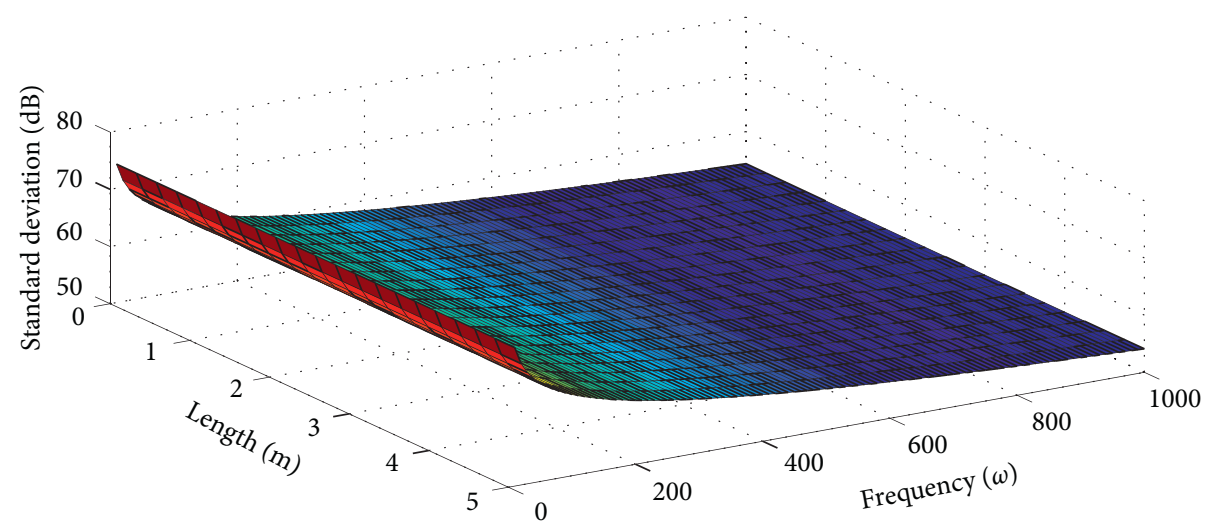

FIgURE 14: Standard deviations of energy densities of the beam. The reference energy density is $10^{-12} \mathrm{~J} / \mathrm{m}^{2}$.

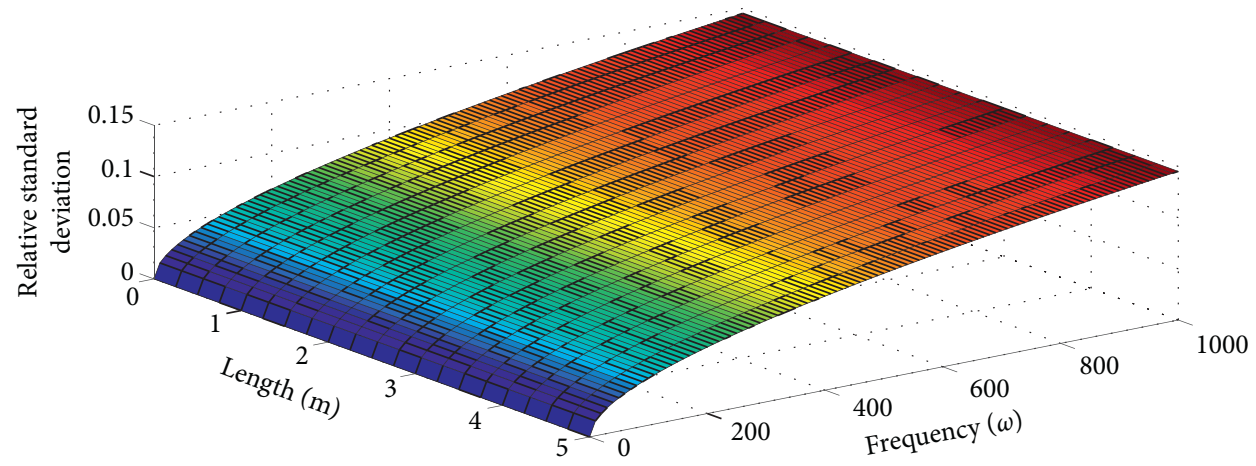

FIgURE 15: Relative standard deviations of energy densities of the beam. 
for the beam is more significant in higher frequencies and remains at a steady level in space at a fixed frequency. And the rate of growth becomes smaller in higher frequencies which differ from that for the rod.

\section{Conclusions}

Vibration prediction of many practical structures demands more on vibration research due to structural complexity and high-frequency analysis. As an approach to high-frequency responses, EFA is now widely applied to many engineering fields. In this work, a general method was presented for predicting the energy density responses. Based on the Green kernels of vibrating rods and beams, the method provided new formulations for rods and beams using the summation of propagating waves. The advantage of the proposed method is that it provides a systematic way to analyse energy responses of structures with analytical formulations and enabled direct solutions of energy density for vibrating structures which promotes the analysis of vibration in energy view. With the proposed method, formulations of energy densities are derived for rods and beams with probabilistic parameters. Simulations for a random-location excitation case were performed to validate the formulations. The proposed method provides an approach to predict energy density responses analytically which facilitates vibration energy analysis of structures. And the extension of the method to structures with an uncertain parameter of other types is the subject of future research.

\section{Data Availability}

The data used to support the findings of this study are available from the corresponding author upon request.

\section{Conflicts of Interest}

The authors declare that there are no conflicts of interest regarding the publication of this paper.

\section{Acknowledgments}

This work was funded by Ph. D funding of NCIAE (BKY2020-12).

\section{References}

[1] R. H. Lyon and G. Maidanik, "Power flow between linearly coupled oscillators," The Journal of the Acoustical Society of America, vol. 34, no. 5, pp. 623-639, 1962.

[2] R. H. Lyon and E. Eichler, "Random vibration of connected structures," The Journal of the Acoustical Society of America, vol. 36, no. 7, pp. 1344-1354, 1964.

[3] R. H. Lyon, Statistical Energy Analysis of Dynamical Systems: Theory and Applications, Cambridge MIT Press, Cambridge, MA, USA, 1975.

[4] E. P. B. Reynders and R. S. Langley, "Cross-frequency and band-averaged response variance prediction in the hybrid deterministic-statistical energy analysis method," Journal of Sound and Vibration, vol. 428, pp. 119-146, 2018.

[5] G. Petrone, A. G. Melillo, and S. De Rosa, "A statistical energy analysis (SEA) model of a fuselage section for the prediction of the internal sound pressure level (SPL) at cruise flight conditions," Aerospace Science and Technology, vol. 88, pp. 340349, 2019.

[6] Q. Chen, Y. Q. Fei, and X. S. YangWu, "Prediction of the transient energy response for complex vibro-acoustic systems," Journal of Mechanical Science and Technology, vol. 33, no. 2, pp. 495-504, 2019.

[7] F. A. Fazzolari and P. Tan, "A hybrid finite element-statistical energy analysis approach for the dynamic response of built-up systems with nonlinear joints," Journal of Sound and Vibration, vol. 2020, Article ID 115696, 2020.

[8] H. Song, Z. Chen, and J. Zhang, "Sensitivity analysis of statistical energy analysis models based on interval perturbation approach," Acta Mechanica, vol. 231, no. 10, pp. 3989-4001, 2020.

[9] V. D. Belov and S. A. Rybak, "Applicability of the transport equation in the one-dimensional wave-propagation problem," Journal of Soviet Physics and Acoustics, vol. 21, no. 2, pp. 110-114, 1975.

[10] V. D. Belov, S. A. Rybak, and B. D. Tartakovskii, "Propagation of vibrational energy in absorbing structures," Journal of Soviet Physics and Acoustic, vol. 23, no. 2, pp. 115-119, 1977.

[11] D. J. Nefske and S. H. Sung, "Power flow finite element analysis of dynamic systems: basic theory and application to beams," Journal of Vibration and Acoustics, vol. 111, no. 1, pp. 94-100, 1989.

[12] J. C. Wohlever and R. J. Bernhard, "Energy distributions in rods and beams," in Proceedings of the AIAA 12th Aeroacoustics Conference, San Antonio, TX, USA, April 1989.

[13] O. M. Bouthier and R. J. Bernhard, "Models of space-averaged energetics of plates," AIAA Journal, vol. 30, no. 3, pp. 616-623, 1992.

[14] O. M. Bouthier and R. J. Bernhard, "Simple models of the energetics of transversely vibrating plates," Journal of Sound and Vibration, vol. 182, no. 1, pp. 149-164, 1995.

[15] D.-H. Park, S.-Y. Hong, H.-G. Kil, and J.-J. Jeon, "Power flow models and analysis of in-plane waves in finite coupled thin plates," Journal of Sound and Vibration, vol. 244, no. 4, pp. 651-668, 2001.

[16] Y.-H. Park and S.-Y. Hong, "Vibrational energy flow analysis of corrected flexural waves in Timoshenko beam - Part I: theory of an energetic model," Shock and Vibration, vol. 13, no. 3, pp. 137-165, 2006.

[17] Y. H. Park and S. Y. Hong, "Vibrational power flow models for transversely vibrating finite Mindlin plate," Journal of Sound \& Vibration, vol. 317, no. 3-5, pp. 800-840, 2008.

[18] J.-B. Han, S.-Y. Hong, J.-H. Song, and H.-W. Kwon, "Vibrational energy flow models for the Rayleigh-Love and Rayleigh-Bishop rods," Journal of Sound and Vibration, vol. 333, no. 2, pp. 520-540, 2014.

[19] Z. Chen, Z. Yang, N. Guo, and G. Zhang, “An energy finite element method for high frequency vibration analysis of beams with axial force," Applied Mathematical Modelling, vol. 61, pp. 521-539, 2018.

[20] P. E. Cho, Energy Flow Analysis of Coupled Structures, Purdue University, West Lafayette, Indiana, 1993.

[21] F. Bitsie, The Structural-Acoustic Energy Finite Element Method, Purdue University, West Lafayette, Indiana, 1996.

[22] W. Zhang, A. Wang, and N. Vlahopoulos, "An alternative energy finite element formulation based on incoherent orthogonal waves and its validation for marine structures," Finite Elements in Analysis and Design, vol. 38, no. 12, pp. 1095-1113, 2002. 
[23] W. Zhang, A. Wang, N. Vlahopoulos, and K. Wu, "Highfrequency vibration analysis of thin elastic plates under heavy fluid loading by an energy finite element formulation," Journal of Sound and Vibration, vol. 263, no. 1, pp. 21-46, 2003.

[24] W. Zhang, A. Wang, and N. Vlahopoulos, "A vibration analysis of stiffened plates under heavy fluid loading by an energy finite element analysis formulation," Finite Elements in Analysis and Design, vol. 41, no. 11-12, pp. 1056-1078, 2005.

[25] H.-W. Kwon, S.-Y. Hong, and J.-H. Song, "Vibrational energy flow analysis of coupled cylindrical thin shell structures," Journal of Mechanical Science and Technology, vol. 30, no. 9, pp. 4049-4062, 2016.

[26] Z. Lin, X. Chen, and B. Zhang, "Application of the energy finite element analysis to vibration of beams with stepped thickness and variable cross-section," Journal of Vibroengineering, vol. 20, no. 6, pp. 2237-2252, 2018.

[27] Z. Liu, J. Niu, and X. Gao, “An improved approach for analysis of coupled structures in Energy Finite Element Analysis using the coupling loss factor," Computers \& Structures, vol. 210, pp. 69-86, 2018.

[28] H. M. Navazi, A. Nokhbatolfoghahaei, Y. Ghobad, and H. Haddadpour, "Experimental measurement of energy density in a vibrating plate and comparison with energy finite element analysis," Journal of Sound and Vibration, vol. 375, pp. 289-307, 2016.

[29] M. Shinozuka and C.-M. Jan, "Digital simulation of random processes and its applications," Journal of Sound and Vibration, vol. 25, no. 1, pp. 111-128, 1972.

[30] R. S. Langley and A. W. M. Brown, "The ensemble statistics of the energy of a random system subjected to harmonic excitation," Journal of Sound and Vibration, vol. 275, no. 3-5, pp. 823-836, 2004.

[31] C. Soize, "A model and numerical method in the medium frequency range for vibroacoustic predictions using the theory of structural fuzzy," The Journal of the Acoustical Society of America, vol. 94, no. 2, pp. 849-865, 1993.

[32] Z. Qiu, S. Chen, and J. Na, "The Rayleigh quotient method for computing eigenvalue bounds of vibrational systems with interval parameters," Acta Mechanica Solida Sinica, vol. 6, pp. 309-318, 1993.

[33] N. Vu-Bac, T. Lahmer, X. Zhuang, T. Nguyen-Thoi, and T. Rabczuk, "A software framework for probabilistic sensitivity analysis for computationally expensive models," Advances in Engineering Software, vol. 100, pp. 19-31, 2016.

[34] F. Fahy and J. Walker, Advanced Applications in Acoustics, Noise and Vibration, Spon Press, London, UK, 2004.

[35] F. Fahy and P. Gardonio, Sound and Structural Vibration: Radiation, Transmission and Response, Elsevier, Oxford, UK, 2nd edition, 2007. 